PROCEEDINGS OF THE

AMERICAN MATHEMATICAL SOCIETY

Volume 137, Number 10, October 2009, Pages 3329-3334

S 0002-9939(09)09815-3

Article electronically published on June 5, 2009

\title{
ESSENTIALLY SPECTRALLY BOUNDED LINEAR MAPS
}

\author{
M. BENDAOUD AND A. BOURHIM
}

(Communicated by Nigel J. Kalton)

\begin{abstract}
Let $\mathcal{L}(\mathcal{H})$ be the algebra of all bounded linear operators on an infinite dimensional complex Hilbert space $\mathcal{H}$. We characterize essentially spectrally bounded linear maps from $\mathcal{L}(\mathcal{H})$ onto $\mathcal{L}(\mathcal{H})$ itself. As a consequence, we characterize linear maps from $\mathcal{L}(\mathcal{H})$ onto $\mathcal{L}(\mathcal{H})$ itself that compress different essential spectral sets such as the the essential spectrum, the (left, right) essential spectrum, and the semi-Fredholm spectrum.
\end{abstract}

\section{Introduction AND STATEMENT OF THE MAIN RESUlt}

Throughout this paper, $\mathcal{H}$ will denote an infinite dimensional complex Hilbert space and $\mathcal{L}(\mathcal{H})$ will denote the algebra of all bounded linear operators on $\mathcal{H}$. The closed ideal of all compact operators on $\mathcal{H}$ is denoted by $\mathcal{K}(\mathcal{H})$, and the Calkin algebra is denoted, as usual, by $\mathcal{C}(\mathcal{H}):=\mathcal{L}(\mathcal{H}) / \mathcal{K}(\mathcal{H})$. For an operator $T \in \mathcal{L}(\mathcal{H})$, let $\sigma_{e}(T), \sigma_{l e}(T), \sigma_{r e}(T)$, and $\sigma_{S F}(T)$ denote the essential spectrum, the left essential spectrum, the right essential spectrum, and the semi-Fredholm spectrum, respectively, of $T$; see for instance 11. The essential norm of the operator $T$ is given by $\|T\|_{e}:=\operatorname{dist}(T, \mathcal{K}(\mathcal{H}))$, and the essential spectral radius, denoted by $r_{e}(T)$, is the limit of the convergent sequence $\left(\left\|T^{n}\right\|_{e^{1 / n}}\right)_{n \geq 1}$. It coincides with $r(\pi(T))$ the classical spectral radius of $\pi(T)$, where $\pi$ denotes the canonical quotient map from $\mathcal{L}(\mathcal{H})$ onto $\mathcal{C}(\mathcal{H})$

New contributions to the study of linear preserver problems in $\mathcal{L}(\mathcal{H})$ have recently been made in 4, 5, 9, 20, 21, 22]. In [22], Mbekhta has treated the problem of characterizing surjective linear maps on $\mathcal{L}(\mathcal{H})$ preserving the set of Fredholm operators in both directions. He proved, in particular, that a surjective linear map on $\mathcal{L}(\mathcal{H})$ preserves the set of Fredholm operators in both directions if and only if it leaves invariant the closed ideal $\mathcal{K}(\mathcal{H})$ of all compact operators and the induced map on the Calkin algebra, $\mathcal{C}(\mathcal{H}):=\mathcal{L}(\mathcal{H}) / \mathcal{K}(\mathcal{H})$, is either an automorphism or an antiautomorphism multiplied by an invertible element in $\mathcal{C}(\mathcal{H})$. In [9], Cui and Hou

Received by the editors June 23, 2008.

2000 Mathematics Subject Classification. Primary 47B49; Secondary 47A10, 47D25.

Key words and phrases. Linear preserver problem, spectrally bounded linear map, essential spectral radius, Fredholm and semi-Fredholm operators.

The second author was supported by an adjunct professorship at Laval University. 
established independently similar results and characterized linear maps on $\mathcal{L}(\mathcal{H})$ preserving certain essential spectral sets such as the set of (left, right) Fredholm operators and the set of semi-Fredholm operators. Part of [21] is also devoted to the study of surjective linear maps on $\mathcal{L}(\mathcal{H})$ preserving the set of semi-Fredholm operators in both directions, and analogous results are obtained.

It should be noted that [20,21] contain many good ideas and elegant results which opened the way for certain authors to generalize the results from [9, 20, 21, 22. In 4, Bendaoud, Bourhim and Sarih presented one main result with a simple proof which characterizes surjective linear maps on $\mathcal{L}(\mathcal{H})$ preserving the essential spectral radius and recaptured, as consequences, the main results of $[9,20,21,22$. While in [5], Boudi and Hadder presented several main results on surjective linear maps on $\mathcal{L}(\mathcal{H})$ preserving, in one direction, generalized invertibility, and Fredholm and semiFredholm operators. As a consequence of their main results, they also recaptured the results of $[9,20,21,22$. However, neither the main result of [4] nor the main results of [5] can be deduced from each other.

In this paper, we unify and extend all the results from [4, 5, 9, 20, 21, 22, by a characterization of surjective essentially spectrally bounded linear maps on $\mathcal{L}(\mathcal{H})$. Recall that a linear map $\phi$ from $\mathcal{L}(\mathcal{H})$ into itself is said to be surjective up to compact operators if $\mathcal{L}(\mathcal{H})=\operatorname{range}(\phi)+\mathcal{K}(\mathcal{H})$, and it is called essentially spectrally bounded if there exists a positive constant $M$ such that $r_{e}(\phi(T)) \leq M r_{e}(T)$ for all $T \in \mathcal{L}(\mathcal{H})$. This paper presents a complete characterization of such maps. Its proof below is considerably simpler than the ones given in [5, 9, 20, 21, 22, for closely related results.

Theorem 1.1. Assume that $\phi \mathcal{L}(\mathcal{H}) \rightarrow \mathcal{L}(\mathcal{H})$ is a linear map which is surjective up to compact operators. Then $\phi$ is essentially spectrally bounded if and only if $\phi(\mathcal{K}(\mathcal{H})) \subseteq \mathcal{K}(\mathcal{H})$ and the induced map $\widehat{\phi}: \mathcal{C}(\mathcal{H}) \rightarrow \mathcal{C}(\mathcal{H})$ defined by $\widehat{\phi}(\pi(T)):=$ $\pi(\phi(T))(T \in \mathcal{L}(\mathcal{H}))$ is either a continuous epimorphism or a continuous antiepimorphism multiplied by a nonzero scalar.

Of course the "if" part is obvious. For the "only if" part, we first use Vesentini's theorem, on subharmonity of the spectral radius when composed with a holomorphic function, together with Liouville's theorem to show that if $\phi$ is a surjective essentially spectrally bounded linear map on $\mathcal{L}(\mathcal{H})$, then $\phi$ leaves invariant the ideal of all compact operators on $\mathcal{H}$, and therefore the induced mapping on the Calkin algebra $\mathcal{C}(\mathcal{H})$ is a well defined spectrally bounded linear map. To conclude the proof, we apply Lemma 2.1. which is a variant of several results that characterize spectrally bounded linear maps from a purely infinite $C^{*}$-algebra with real rank zero onto a semisimple Banach algebra; see $7,10,12,15,16,17,18,19,24$.

It is very well known that the Calkin algebra $\mathcal{C}(\mathcal{H})$ is a simple algebra provided that $\mathcal{H}$ is a separable Hilbert space; see for instance [8. In this case, every epimorphism (resp. anti-epimorphism) on $\mathcal{C}(\mathcal{H})$ is an automorphism (resp. antiautomorphism). It should also be noted that, unlike $\mathcal{L}(\mathcal{H})$, the Calkin algebra $\mathcal{C}(\mathcal{H})$ has, in general, outer automorphisms as shown by Phillips and Weaver [23, who answered negatively a long-standing problem which asks whether every automorphism of $\mathcal{C}(\mathcal{H})$ is inner. However, Farah showed in [11 that there are complex Hilbert spaces $\mathcal{H}$ such that all the automorphisms of the corresponding Calkin algebra $\mathcal{C}(\mathcal{H})$ are inner. 


\section{Proof of the main Result}

In this section, we first state and prove the promised auxiliary lemma which will be needed for the proof of Theorem 1.1. After proving the main result, we present some consequences which were discovered in [5].

A linear map $\phi: \mathcal{A} \rightarrow \mathcal{B}$ between two unital Banach algebras $\mathcal{A}$ and $\mathcal{B}$ is called unital if $\phi(\mathbf{1})=\mathbf{1}$, and it is said to be a Jordan homomorphism if $\phi\left(a^{2}\right)=\phi(a)^{2}$ for all $a \in \mathcal{A}$. Equivalently, the map $\phi$ is a Jordan homomorphism if and only if $\phi(a b+b a)=\phi(a) \phi(b)+\phi(b) \phi(a)$ for all $a$ and $b$ in $\mathcal{A}$. It is called a Jordan isomorphism provided that it is a bijective Jordan homomorphism. Clearly, every homomorphism and every anti-homomorphism is a Jordan homomorphism. Recall also that the map $\phi$ is called spectrally bounded if there exists a constant $M>0$ such that $r(\phi(a)) \leq M r(a)$ holds for every $a \in \mathcal{A}$, where $r($.$) denotes, as usual, the$ spectral radius.

Recall that a $C^{*}$-algebra $\mathcal{A}$ is said to have real rank zero if the set of all real combinations of orthogonal projections is dense in the set of all selfadjoint elements of $\mathcal{A}$. It said to be purely infinite if it has no characters and if for every pair of positive elements $a$ and $b$ in $\mathcal{A}$ with $a \in \overline{\mathcal{A} b \mathcal{A}}$ there is a sequence $\left(x_{n}\right)_{n \in \mathbb{N}}$ in $\mathcal{A}$ such that $a=\lim _{n} x_{n}^{*} b x_{n}$; see [14. Denote by $\sim$ the usual Murray-von Neumann equivalence relation on the set of all projections of $\mathcal{A}$, and recall that a nonzero projection $p$ in $\mathcal{A}$ is said to be properly infinite if there exist mutually orthogonal subprojections $p_{1}$ and $p_{2}$ of $p$ such that $p_{1} \sim p \sim p_{2}$. If the identity of $\mathcal{A}$ is properly infinite, then $\mathcal{A}$ itself is called properly infinite.

Lemma 2.1. Let $\mathcal{A}$ be a unital purely infinite $C^{*}$-algebra with real rank zero and let $\mathcal{B}$ is a semisimple unital Banach algebra. If $\varphi: \mathcal{A} \rightarrow \mathcal{B}$ is a surjective spectrally bounded linear map, then there exist a central invertible element $c$, viz., $\varphi(\mathbf{1})$, and a Jordan epimorphism $J: \mathcal{A} \rightarrow \mathcal{B}$ such that $\varphi(x)=c J(x)$ for all $x \in \mathcal{A}$.

Unlike [17, Theorem 3.6 2.5], [19, Theorem B] and [15, Corllary 2.5], we do not assume that $\varphi$ is unital, which is new and which is, in fact, needed for our purposes. However, the proof of this lemma is on the straightforward side and is included here for the sake of completeness.

Proof of Lemma 2.1. Let $p$ be a nonzero projection in $\mathcal{A}$ and note that, since $\mathcal{A}$ is purely infinite, $p$ is properly infinite; see [14, Theorem 4.16]. Thus $p \mathcal{A} p$ is a properly infinite $C^{*}$-algebra, and each of its elements can be written as a finite sum of elements of $p \mathcal{A} p$ with square zero; see [15, proposition 2.1]. By [17, Lemma 3.3], we have

$$
\varphi(p) \varphi(q)+\varphi(q) \varphi(p)=0
$$

for all projections $q \in \mathcal{A}$ which are orthogonal to $p$. Now, apply (2.1) to $p$ and $q=\mathbf{1}-p$ to see that

$$
\varphi(p) \varphi(\mathbf{1})+\varphi(\mathbf{1}) \varphi(p)=2(\varphi(p))^{2}
$$

Multiplying this identity separately on the left and then on the right by $\varphi(p)$ and comparing the obtained equations, we get that

$$
\varphi(\mathbf{1})(\varphi(p))^{2}=(\varphi(p))^{2} \varphi(\mathbf{1})
$$


Now, let $a=\sum_{i=1}^{i=n} \lambda_{i} p_{i}$ be a linear combination of orthogonal projections $p_{1}, \ldots, p_{n}$ of $\mathcal{A}$. In view of (2.1), we have $\varphi(a)^{2}=\sum_{i=1}^{i=n} \lambda_{i}^{2}\left(\varphi\left(p_{i}\right)\right)^{2}$ and $(\varphi(a))^{2} \varphi(\mathbf{1})=$ $\varphi(\mathbf{1})(\varphi(a))^{2}$ by (2.3). As $\varphi$ is continuous (see [2, Theorem 5.5.2]) and $\mathcal{A}$ has real rank zero, we get that $(\varphi(h))^{2} \varphi(\mathbf{1})=\varphi(\mathbf{1})(\varphi(h))^{2}$ for all selfadjoint elements $h \in \mathcal{A}$. From this, it follows easily that $(\varphi(x))^{2} \varphi(\mathbf{1})=\varphi(\mathbf{1})(\varphi(x))^{2}$ for all $x \in \mathcal{A}$. Since every element $y$ in $\mathcal{B}$ can be written as a finite sum of square elements in $\mathcal{B}$ and $\varphi$ is surjective, we see that $\varphi(\mathbf{1})$ is a central element of $\mathcal{B}$. Therefore, it follows from (2.2) that

$$
(\varphi(p))^{2}=\varphi(p) \varphi(\mathbf{1})=\varphi\left(p^{2}\right) \varphi(\mathbf{1})
$$

for all projections $p \in \mathcal{A}$. Just as before and employing (2.4) instead of (2.3), we get that $(\varphi(x))^{2}=\varphi\left(x^{2}\right) \varphi(\mathbf{1})$ for all $x \in \mathcal{A}$. As $\varphi$ is surjective, there is an element $u \in \mathcal{A}$ such that $\varphi(u)=\mathbf{1}$, and $\mathbf{1}=(\varphi(u))^{2}=\varphi\left(u^{2}\right) \varphi(\mathbf{1})=\varphi(\mathbf{1}) \varphi\left(u^{2}\right)$. This shows that $\varphi(\mathbf{1})$ is invertible.

Finally, set $J(x):=\varphi(\mathbf{1})^{-1} \varphi(x)(x \in \mathcal{A})$, and note that

$$
(J(x))^{2}=\varphi(\mathbf{1})^{-2}(\varphi(x))^{2}=\varphi(\mathbf{1})^{-1} \varphi\left(x^{2}\right)=J\left(x^{2}\right)
$$

for all $x \in \mathcal{A}$. Thus $J$ is a Jordan epimorphism, and the proof is therefore complete.

We are now in a position to prove the main result of this paper.

Proof of Theorem 1.1. Assume that there is a positive constant $M$ such that $r_{e}(\phi(T)) \leq M r_{e}(T)$ for all $T \in \mathcal{L}(\mathcal{H})$, and let us establish the "only if" part of Theorem 1.1. We first show that $\phi$ leaves $\mathcal{K}(\mathcal{H})$ invariant. So pick a compact operator $K \in \mathcal{K}(\mathcal{H})$, and let us prove that $\phi(K)$ is compact as well. Let $S \in \mathcal{L}(\mathcal{H})$ be an arbitrary operator on $\mathcal{H}$ and note that, since $\phi$ is surjective up to compact operators, there exist $T \in \mathcal{L}(\mathcal{H})$ and $K_{0} \in \mathcal{K}(\mathcal{H})$ such that $S=\phi(T)+K_{0}$. For every $\lambda \in \mathbb{C}$, we have

$$
\begin{aligned}
r(\lambda \pi(\phi(K))+\pi(S)) & =r(\pi(\lambda \phi(K)+S))=r_{e}(\lambda \phi(K)+S) \\
& =r_{e}\left(\phi(\lambda K+T)+K_{0}\right)=r_{e}(\phi(\lambda K+T)) \\
& \leq M r_{e}(\lambda K+T)=M r_{e}(T) .
\end{aligned}
$$

Since $\lambda \mapsto r(\lambda \pi(\phi(K))+\pi(S))$ is a subharmonic function on $\mathbb{C}$, Liouville's Theorem implies that $r(\pi(\phi(K))+\pi(S))=r(\pi(S))$. As $S$ is arbitrary in $\mathcal{L}(\mathcal{H})$, it follows from semi-simplicity of $\mathcal{C}(\mathcal{H})$ and the Zemánek's characterization of the radical [2, Theorem 5.3.1] that $\pi(\phi(K))=0$ and $\phi(K) \in \mathcal{K}(\mathcal{H})$.

Therefore $\phi(\mathcal{K}(\mathcal{H})) \subseteq \mathcal{K}(\mathcal{H})$, and $\phi$ induces a surjective spectrally bounded linear map $\widehat{\phi}: \mathcal{C}(\mathcal{H}) \rightarrow \mathcal{C}(\mathcal{H})$ defined by $\widehat{\phi}(\pi(T)):=\pi(\phi(T))$ for all $T \in \mathcal{L}(\mathcal{H})$. As $\mathcal{C}(\mathcal{H})$ is a purely infinite $C^{*}$-algebra with real rank zero, Lemma 2.1 tells us that $\widehat{\phi}$ is a continuous Jordan epimorphism $J$ multiplied by an invertible central element of $\mathcal{C}(\mathcal{H})$, viz., $\widehat{\phi}(\pi(\mathbf{1}))$. However, since the centre of $\mathcal{C}(\mathcal{H})$ is trivial, $\widehat{\phi}(\pi(\mathbf{1}))$ must be a nonzero complex number $c$. As $\mathcal{C}(\mathcal{H})$ is prime, the well known theorem of Herstein [13. tells us that $J$ must be an epimorphism or an anti-epimorphism. The proof is therefore complete.

As a consequence, we describe linear maps compressing certain essential spectral sets. Recall that a linear map $\phi: \mathcal{L}(\mathcal{H}) \rightarrow \mathcal{L}(\mathcal{H})$ is said to compress the essential spectrum if $\sigma_{e}(\phi(T)) \subseteq \sigma_{e}(T)$ for all $T \in \mathcal{L}(\mathcal{H})$. The linear maps compressing the 
left essential spectrum, the right essential spectrum, or semi-Fredholm spectrum are defined in a similar way.

Corollary 2.2. Let $\phi: \mathcal{L}(\mathcal{H}) \rightarrow \mathcal{L}(\mathcal{H})$ be a linear map surjective up to compact operators. Then $\phi$ compresses the essential spectrum (the semi-Fredholm spectrum) if and only if $\phi(\mathcal{K}(\mathcal{H})) \subseteq \mathcal{K}(\mathcal{H})$ and the induced map $\widehat{\phi}: \mathcal{C}(\mathcal{H}) \rightarrow \mathcal{C}(\mathcal{H})$ is either a continuous epimorphism or a continuous anti-epimorphism.

Proof. It suffices to prove the "only if" part. Assume that $\phi$ compresses the essential spectrum (the semi-Fredholm spectrum), and note that, in this case, $\phi$ is an essentially spectrally bounded linear map. By Theorem 1.1, $\phi(\mathcal{K}(\mathcal{H})) \subseteq \mathcal{K}(\mathcal{H})$, and the induced map $\widehat{\phi}: \mathcal{C}(\mathcal{H}) \rightarrow \mathcal{C}(\mathcal{H})$ is either a continuous epimorphism or a continuous anti-epimorphism multiplied by a nonzero scalar $c$. Of course, $c$ is equal to 1 because $\{c\}=\sigma_{e}(\phi(\mathbf{1})) \subset \sigma_{e}(\mathbf{1})=\{1\}$.

The proof of the other case when $\phi$ compresses the semi-Fredholm spectrum holds in a similar way.

The second consequence characterizes surjective linear maps on $\mathcal{L}(\mathcal{H})$ compressing the left and right essential spectrum. Its proof proceeds along the same lines as the one for the above corollary.

Corollary 2.3. Let $\phi: \mathcal{L}(\mathcal{H}) \rightarrow \mathcal{L}(\mathcal{H})$ be a linear map surjective up to compact operators. Then $\phi$ compresses the left essential spectrum (the right essential spectrum) if and only if $\phi(\mathcal{K}(\mathcal{H})) \subseteq \mathcal{K}(\mathcal{H})$ and the induced map $\widehat{\phi}: \mathcal{C}(\mathcal{H}) \rightarrow \mathcal{C}(\mathcal{H})$ is a continuous epimorphism.

We finally close this paper with the following remark.

Remark 2.4. As in [3, Section 5] and [6, Section 4], the main result of this paper and its corollaries can be stated in a more general situation where one considers essentially spectrally bounded linear maps from a unital purely infinite $C^{*}$-algebra with real rank zero onto a semisimple Banach algebra.

\section{ACKNOWLEDGMENTS}

The authors thank Professor M. Brešar very much for his comments and remarks which led to the final version of this paper. They also thank the organizers of "The 5th Linear Algebra Workshop, Kranjska Gora, Slovenia (May 27 - June 5, 2008)" and "The 2nd International Conference on Linear Preservers and Operator Theory, Meknès, Morocco (June 2-6, 2008)", where the main result of this paper was announced.

\section{REFERENCES}

[1] P. Aiena, Fredholm and local spectral theory, with applications to multipliers, Kluwer Academic Publishers, 2004. MR2070395 (2005e:47001)

[2] B. Aupetit, A primer on spectral theory, Springer-Verlag, New York, 1991. MR.1083349 (92c:46001)

[3] M. Bendaoud, A. Bourhim, M. Burgos and M. Sarih, Linear maps preserving Fredholm and Atkinson elements of $C^{*}$-algebras, Linear and Multilinear Alg., to appear.

[4] M. Bendaoud, A. Bourhim and M. Sarih, Linear maps preserving the essential spectral radius, Linear Algebra Appl., 428(2008) 1041-1045. MR2382111 (2008m:47051)

[5] N. Boudi and Y. Hadder, On linear maps preserving generalized invertibility and related properties, J. Math. Anal. Appl., 345(2008), no. 1, 20-25. MR.2422630 (2009e:47058)

[6] A. Bourhim and M. Burgos, Linear maps preserving the minimum modulus, submitted. 
[7] M. Brešar and P. Šemrl, Linear maps preserving the spectral radius, J. Funct. Anal., 142(1996), no. 2, 360-368. MR1423038 (97i:47070)

[8] J.W. Calkin, Two-sided ideals and congruences in the ring of bounded operators in Hilbert space, Ann. of Math. (2), 42(1941) 839-873. MR0005790(3:208c)

[9] J. Cui and J. Hou, Linear maps preserving essential spectral functions and closeness of operator ranges, Bull. London Math. Soc., 39(2007) 575-582. MR2346937 (2008i:47079)

[10] J. Cui and J. Hou, The spectrally bounded linear maps on operator algebras, Studia Math., 150(2002), no. 3, 261-271. MR1891847 (2003a:47079)

[11] I. Farah, All automorphisms of the Calkin algebra are inner, arXiv:0705.3085v1.

[12] A. Fošner and P. Šemrl, Spectrally bounded linear maps on $\mathcal{B}(X)$, Canad. Math. Bull., 47(2004), no. 3, 369-372. MR2072597 (2005e:47096)

[13] I.N. Herstein, Jordan homomorphisms, Trans. Amer. Math. Soc., 81(1956) 331-341. MR0076751 (17:938f)

[14] E. Kirchberg and M. Rørdam, Non-simple purely infinite $C^{*}$-algebras, Amer. J. Math., 122(2000) 637-666. MR.1759891(2001k:46088)

[15] Y.-F. Lin and M. Mathieu, Jordan isomorphism of purely infinite $C^{*}$-algebras, Quart. J. Math., 58(2007) 249-253. MR2334865 (2008k:46189)

[16] M. Mathieu and G.J. Schick, First results on spectrally bounded operators, Studia Math., 152(2002), no. 2, 187-199. MR1916549(2003e:47066)

[17] M. Mathieu and G.J. Schick, Spectrally bounded operators from von Neumann algebras, J. Operator Theory, 49(2003) 285-293. MR 1991740 (2004c:47079)

[18] M. Mathieu, Spectrally bounded operators on simple $C^{*}$-algebras. II, Irish Math. Soc. Bulletin, 54(2004) 33-40. MR2138429 (2005m:47074)

[19] M. Mathieu, Spectrally bounded operators on simple $C^{*}$-algebras, Proc. Amer. Math. Soc., 132(2004) 443-446. MR2022367(2004i:47065)

[20] M. Mbekhta, L. Rødman and P. Semrl, Linear maps preserving generalized invertibility, Int. Equ. Operator Theory, 55(2006) 93-109. MR2226639(2007e:47055)

[21] M. Mbekhta and P. Šemrl, Linear maps preserving semi-Fredholm operators and generalized invertibility, Linear Multilinear Alg., 57(2009), no. 1, 55-64.

[22] M. Mbekhta, Linear maps preserving the set of Fredholm operators, Proc. Amer. Math. Soc., 135(2007) 3613-3619. MR2336577 (2008h:47075)

[23] N.C. Phillips and N. Weaver, The Calkin algebra has outer automorphisms, Duke Math. J., 139(2007) 185-202. MR2322680(2009a:46123)

[24] P. Šemrl, Spectrally bounded linear maps on B(H), Quart. J. Math. Oxford Ser. (2), 49(1998) 87-92. MR1617339 (2000b:47090)

Département de Mathématiques, Université Moulay Ismail, Meknès, Morocco

E-mail address: bendaoudmohamed@gmail.com

Département de Mathématiques et de Statistique, Université Laval, Québec G1K 7P4, CANAdA

E-mail address: bourhim@mat.ulaval.ca

Current address: Department of Mathematics, Syracuse University, 215 Carnegie Building, Syracuse, New York 13244

E-mail address: abourhim@syr.edu 